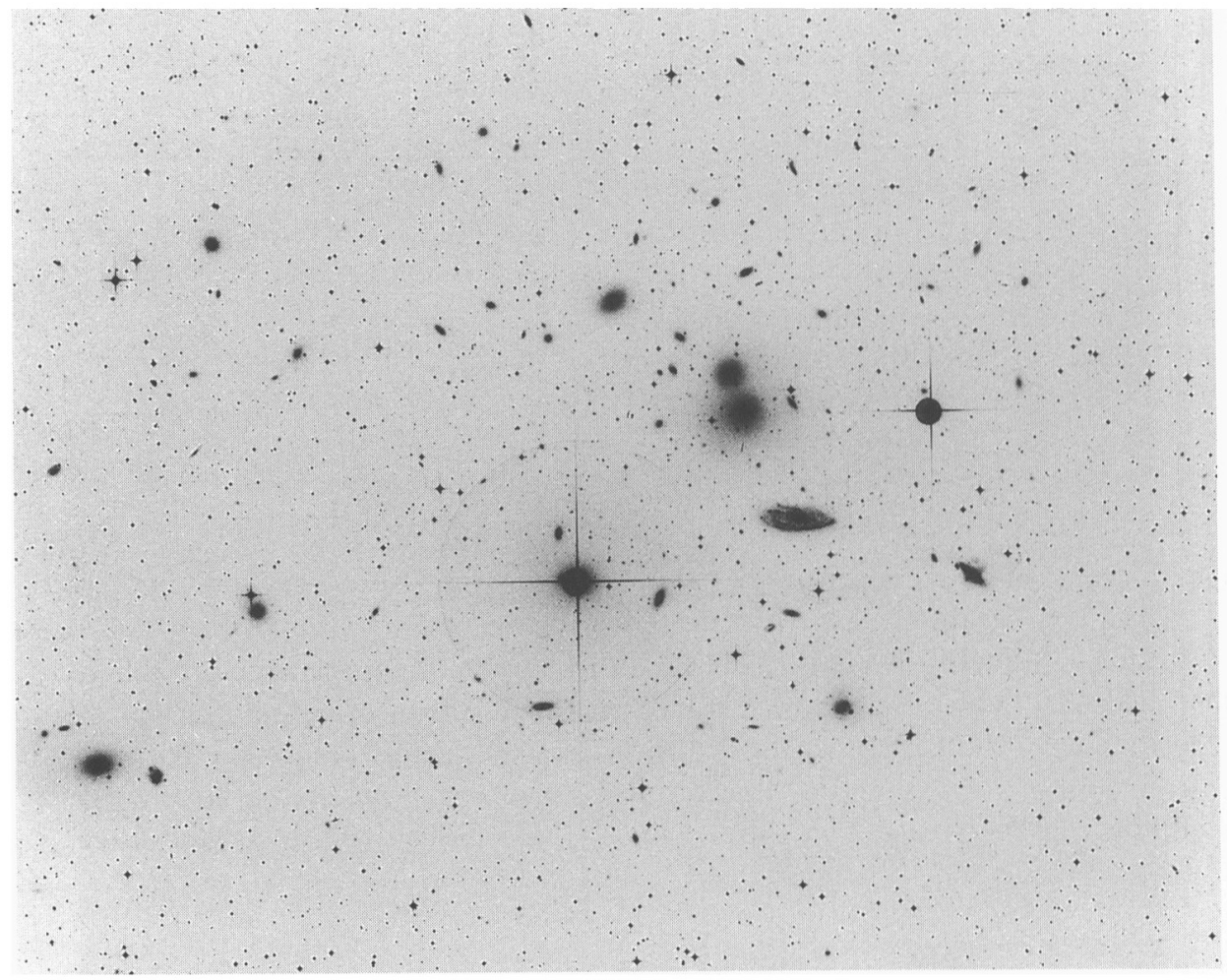

Plate 5: Abell 1060. The photograph shows only the central part of the cluster and covers only a small part of the provided film.

\title{
CLASSIFICATION OF STELLAR SPECTRA
}

\section{M.M. Dworetsky}

University of London Observatory, University College London, Mill Hill Park, London NW7 2QS, U.K.

Summary: The objectives of this exercise are to gain familiarity with the appearance of the spectra of stars, to understand some of the temperature and luminosity criteria used to classify spectra, and to gain insights into the relationship between the appearance of stellar spectra and physical conditions in stellar atmospheres.

Our experience with exercises in which beginning undergraduate astronomy degree students are challenged to classify "real" stellar spectra according to Harvard 
or MK types has not been entirely satisfactory. These inexperienced students lack appreciation of the subtleties involved and are sometimes unable to explain the physical phenomena underlying the appearance of stellar spectra. For this reason, we decided some years ago to construct a "cloudy night" experiment, based on high-quality slit spectrograms taken at the University of London Observatory, which promotes familiarity with the appearance of spectra of different stellar types, and also illustrates the underlying physical basis of spectral classification (ionization and excitation). The equipment required is rudimentary: aside from the set of highquality photographic prints, all that is needed is a good transparent plastic ruler, an identification chart for the Cu-Ar comparison lamp, a table of identified lines in the star $\gamma$ Pegasi (Aller 1949; Aller and Jugaku 1956), some references and background reading (e.g., Zeilik and Smith, 1987; Roy and Clarke 1982), and a calculator for evaluation of the Saha and Boltzmann formulae.

The photographic prints are first-generation enlargements of (mostly) IIIa-J spectrograms of 13 standard stars, taken with an original dispersion of $140 \AA / \mathrm{mm}$ using the Cassegrain spectrograph described by Dworetsky (1980). The widening, resolution and quality of these prints conforms reasonably well with the original MK specifications. The exercise itself is divided into three sections, of which the first two could serve some instructors as independent "mini-experiments" in their own right.

In the first section, students measure the positions of several comparison lines and as many stellar lines in the spectrum of a B3V star (Fig. 1) as they think they can detect. Experience has shown that, if the measurements are made with care, stellar wavelengths can be determined with an accuracy of the order of $1 \AA$, sufficient for the student to identify the visible stellar lines such as $\mathrm{H}, \mathrm{He}, \mathrm{C}$, and $\mathrm{Mg}$. In the second section, students make eye estimates (on a scale from 1 to 10) of the strength of $\mathrm{H} \gamma$ in the seven spectra from B3V to F9V. They calculate, using the Saha and Boltzmann formulae, the population of $\mathrm{H}$ atoms in the $n=2$ state relative to the total number of $\mathrm{H}$ atoms and ions. This ratio, raised to the 0.4 power and scaled so that $I(\max )=10$, should provide a close fit to the estimated strengths of the Balmer lines if the calculation has been correctly carried out (Aller 1963). To assist the students, we provide a table of stellar effective temperatures and electron pressures, as well as a tabulation of the partition function of $\mathrm{H} \mathrm{I}$. In the final part of the experiment, students examine the spectra of $F$ to $M$ stars and study the systematic behavior of several prominent spectral features.

We assess students on their ability to present their results accurately and clearly, and on their answers to "thought" questions that are asked at each stage of the exercise.

Space limitations preclude a full presentation of the written script here, but the Observatory is able to provide a complete set of the photographic prints of spectra, a copy of the script we have prepared for the students, and instructor's notes, at a low cost (at this writing, approximately US $\$ 18$ for the first set and US $\$ 10$ for each additional set.) that covers the printing and postage expenses. This also includes free replacement of lost or damaged prints, future prints of additional spectra, and 
future revisions to the script. A substantial discount is available for additional sets. Enquiries should be addressed to "The Director" of the Observatory at the author's address given above.

\section{References}

Aller, L.H. 1949, Astrophys. J., 109, 244.

Aller, L.H. 1963, Astrophysics: The Atmospheres of the Sun and Stars, Second Ed.

(Ronald Press: New York), 124.

Aller, L.H. and Jugaku, J. 1956, Astrophys. J., 127, 244.

Dworetsky, M.M. 1980, Q. J. Roy. Astr. Soc., 21, 50.

Roy, A.E. and Clarke, D. 1982, Astronomy: Structure of the Universe, Second Ed. (Adam Hilger: Bristol), ch. 7.

Zeilik, M. and Smith, E.v.P. 1987, Introductory Astronomy and Astrophysics, Second Ed. (Saunders: Philadelphia), ch. 13.

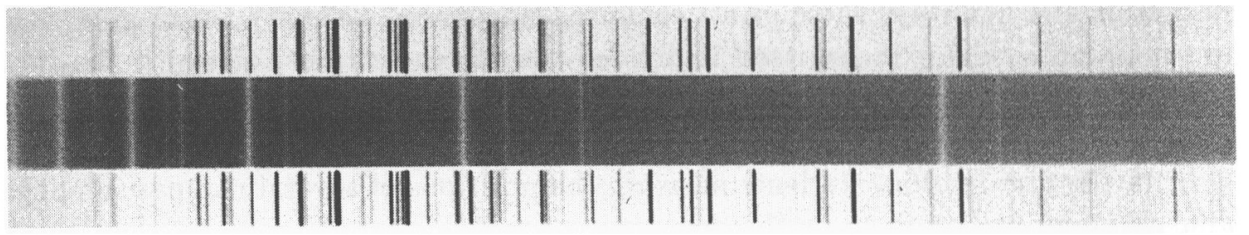

Fig. 1. The spectrogram of the B3V star $\iota$ Herculis used in this experiment (original dispersion $140 \AA / \mathrm{mm}$ ). University of London Observatory photograph.

\section{TELESCOPES FOR STUDENTS}

\section{Roy L. Bishop}

Department of Physics, Acadia University, Wolfville, Nova Scotia, B0P 1X0, Canada

An astronomy class typically is composed of twenty or more students, one instructor, and one telescope. For observing sessions, these numbers mean that at any one moment most of the students are standing, slowly freezing in the dark, waiting their turn at the eyepiece. Moreover, when they do have their 20-second peek, moist breath, a bump by the last observer, or unfamiliarity with the instrument often mean that the object will look hazy, be out of focus, or even be out of view. A couple of sessions under these circumstances can dampen the interest even of keen students. Also, the instructor may be reluctant to let individual students have unsupervised access to the one, expensive, fragile telescope.

Ideally each student would have a telescope during supervised observing sessions. Each telescope should be easy-to-use, rugged, equipped with setting circles, 